\title{
From the Ehlers-Pirani-Schild analysis on the foundations of gravitational theories to extended theories of gravity and dark matter
}

\author{
L. Fatibene \\ Department of Mathematics, University of Torino (Italy) \\ INFN - Sezione di Torino. Iniziativa Specifica Na12 \\ E-mail: lorenzo.fatibenedunito.it

\section{M.Francaviglia*} \\ Department of Mathematics, University of Torino (Italy) \\ INFN - Sezione di Torino. Iniziativa Specifica Na12 \\ E-mail: mauro.francaviglia@unito.it
}

\begin{abstract}
We here investigate how matter can be represented in a Weyl geometry of spacetime typical of Ehlers-Pirani-Schild (EPS) framework. Any timelike field can be regarded as the flow of a fluid and in turn it constrains the gravitational fields which are compatible with that particular fluid. The standard structure of conservation laws for matter leads fix the conformal gauge. The behaviour of Klein-Gordon dynamics under conformal transformations is investigated both at the level of field equations and Lagrangian.
\end{abstract}

Proceedings of the Corfu Summer Institute 2011 School and Workshops on Elementary Particle Physics and Gravity

September 4-18, 2011

Corfu, Greece

\footnotetext{
*Speaker.
} 


\section{Introduction}

During the last decade cosmological and astrophysical precision data seriously questioned the whole standard framework of General Relativity (GR) and its applications to scales much bigger than the solar system. GR is very well tested at solar system scales. All classical tests (deflection of lightrays, precession of perihelia, echo radar delays) thoroughly confirm standard GR based on Hilbert-Einstein dynamics. Also binary pulsar emission rate does confirm precisely standard GR, (despite these are outside the solar system they are in fact star system scale systems as well).

When GR models galactic systems the first mismatch is found. The rotation profile of galaxies shows an anomalous plateau at boundaries which cannot be explained in terms of the gravitational sources which are correlated to light emission. In order to maintain standard GR one is forced to introduce dark sources of the gravitational field (called dark matter) which modify the radial mass profile and make it uncorrelated to the radial light profile. One can phenomenologically shape the distribution of dark matter so that it affects rotation profile and fit the observation. Some theoretical model has been proposed to model dark matter. Despite the lack of direct evidence for dark matter at fundamental level, the model for it meets most reasonable constraints from particle physics. Similar phenomena are encountered at the bigger scale of cluster and superclusters of galaxies.

At cosmological scales, a mismatch between luminosity and Hubble distances of far standard candles (SNIa) as well as correlations among anisotropies of cosmic background radiation (CMB) seem to indicate that recently (in the scale of few billion years) the universe expansion rate has started to accelerate, contrarily to the general expectation which, based on attractive nature of gravity, points to a ever slow expansion rate. Again new gravitational sources are introduced to fit data, this time called dark energy. Currently one of the best models resorts to a small cosmological constant and dark matter. According to this model the standard matter we see in the universe that makes stars, galaxies and clusters is just a small fraction of the gravitational universe out there. Dark matter represents about $30 \%$ of the total matter in the universe, dark energy is about $70 \%$ while stardard matter makes just few percent of the universe.

Moreover, models for dark energy usually produce a number of problems at fundamental level, from instabilities to fine tuning. Furthermore, despite models account quite well for the current observations of the universe, more problematic is defining a dynamics able to account for past and future observations.

A different approach for explaining these observations is to modify the dynamics of the gravitational field. This can be done by considering more general Lagrangians to replace HilbertEinstein Lagrangian and without introducing (or reducing the need of introducing) dark matter or dark energy. A popular class of models considers the Lagrangian $L=\sqrt{g} f(R)$, where $f$ is a generic analytic function of the scalar curvature $R$. One can either assume a Lorentziam metric $g$ of spacetime and consider the purely metric scalar curvature $R(g)$ or assume a metric $g$ and a (usually torsionless) connection $\Gamma$ and consider the metric affine $R(g, \Gamma)=g^{\mu v} R_{\mu v}(\Gamma)$ curvature which depends both on the metric and the connection. The first approach is called purely metric $f(R)$-theories; the second is called metric affine (or Palatini) $f(R)$-theories; see [1], [2], [3].

Then one can choose the function $f$ to fit the observations. The fitting is in some case quite good (as good as for dark matter/dark energy models). Again it is not known if there exists a single choice of $f$ able to account for effects at all scales and at all times. Sometimes fundamental 
problems have been highlighted (again instabilities, renormalization, ...) though these are not showed to be generically present. Modifications of these types of Hilbert-Einstein Lagrangian is also justified as effective theories of string theory and supergravity as well from other approaches to quantum gravity; [3].

However, once one starts to generalize gravitational dynamics it is not clear how to limit modifications and it is quite hard to find properties which are generic in the class of modified theories of gravity; [4], [5]. Even keeping stuck to dynamics based on a generally covariant action principles one has to face a huge class of models which risk to be able to fit anything just in view of the huge number of models available. For example, one can consider geometric invariants other than the scalar curvature, e.g. $R_{\mu v} R^{\mu v}, R_{\alpha \beta \mu v} R^{\alpha \beta \mu v}$ or Weyl tensor invariants. The request of respecting solar system tests puts some constraints but freedom is still huge.

Also, $f(R)$-theories (in both purely metric or metric-affine formulation) are quite naturally described in terms of a new metric $\tilde{g}$ conformally equivalent to the original metric $g$. This usually produces doubts about the physical interpretation of the theory, namely, which is the metric which is used to define distances and clocks? In standard GR there is one canonical metric structure on spacetime so there is not much to discuss. In (Palatini) $f(R)$ theories there is a conformal gauge symmetry which must be fixed somehow to define observational protocols for distances and clocks.

A somehow analogous issue arises about how to couple to matter fields. Minimally coupling is in fact not preserved by conformal transformations since in $f(R)$-theories the conformal factor is determined as a function of the scalar curvature, i.e. $\tilde{g}_{\mu v}=f^{\prime}(R) \cdot g_{\mu v}$ (in dimension $m=4$ ). Accordingly, if matter is minimally coupled to gravitational sector in one conformal gauge, in general it is not in another; see [6], [7]. All these problems point to mismatches in definition of masses and distances of a conformal nature which is precisely what have been first detected as mismatches in new precision observations.

As a matter of fact standard interpretation of standard GR is based on a purely Lorentzian metric. In most models, also using standard Palatini framework, field equations for the connection force the connection to be the Levi-Civita connection of the original metric $g$. On the contrary, in $f(R)$-models the connection is usually forced to be the Levi-Civita connection of another metric $\tilde{g}$ conformal to $g$. With two metrics in the game (luckly enough both defining the same lightcones in spacetime) one has to decide which one (if any) describes free fall of massive test particles.

In the 70s Ehlers-Pirani-Schild (EPS) proposed an axiomatic approach to gravitational theories based on particles and lightrays worldlines (see [8], [9], [10]). In this approach the geometric structure of spacetime is not assumed but it is defined on the congruence of potentially observable worldlines. Hence EPS provides a natural interpretation of spacetime geometry and gravitational physics. As a matter of fact, EPS shows that gravitational systems are naturally described by a conformal class $[g]$ of metrics (which defines lightcones) and a projective class $[\Gamma]$ of connections (which defines free fall of massive particles). The projectively equivalent connections define the same congruence of autoparallel trajectories then the same free fall; accordingly, there is no way to observe a representative of projective class and projective trasformations are gauge transformations; [11].

Of course, the conformal and projective structures have to obey some compatibility condition since they are not expected to be completely unrelated. EPS proposed to assume that null geodesics of the conformal structure (which are conformally invariant unlike the timelike geodesics) are a 
subset of autoparallel congruence of the projective structure. In view of the compatibility condition then the projective gauge can be canonically fixed to define a Weyl geometry $(M,[g], \Gamma)$ on spacetime where

$$
\Gamma_{\beta \mu}^{\alpha}=\{g\}_{\beta \mu}^{\alpha}+\left(g^{\alpha \varepsilon} g_{\beta \mu}-2 \delta_{(\beta}^{\alpha} \delta_{\mu)}^{\varepsilon}\right) A_{\varepsilon}
$$

for some covector $A_{\varepsilon}$, which can be thought to parametrize extra degrees of freedom other then the metric ones. Standard GR is recovered for $A_{\varepsilon}=0$. General Weyl geometries are known to be affected by holonomy problems (length of rules does in general depend on the path). A particular class of Weyl geometries which do not exhibit these holonomic problems are metric Weyl geometries in which there exists a metric $\tilde{g}$ which represents both the conformal structure and the connection, i.e. $\Gamma=\{\tilde{g}\}$. This is more general than standard GR, still there is single metric $\tilde{g}$ which both determines lightcones and free fall. Metric Weyl geometries corresponds to exact covectors $A=d \ln \Phi(x)$ where $\Phi$ is in fact related to the conformal transformation connecting $g$ and $\tilde{g}$.

Metric Weyl geometries represent a natural extension of Hilbert-Einstein gravity. Although there is nothing in EPS really constraining Weyl geometries to be metric if one considers $f(R)$ theories in metric affine formulation then (for matter coupling to $g$ and not to $\Gamma$ ) field equations force the spacetime geometry to be described by a metric Weyl geometry, though in general not by standard GR; see [9], [10].

If matter is allowed to couple to the connection a more general situation is obtained in which more general (non-metric) Weyl geometries can be enforced by field equations; see [7], [4], [5]. To the best of our knowledge not much is known for a generic modification of gravitational theory; when other invariants or generic coupling between matter an connection are allowed dynamics will fail to enforce EPS compatibility between conformal and projective structure, with all interpretation and fundational problems one can imagine to show up in these cases.

One should not need to remark that electromagnetic field is deeply entangled with our protocols for observation. We rely on the properties of electromagnetic field to measure distances and to build clocks. All physical experiments use electromagnetic field in quite a peculiar way. Although this issue was usually overlooked (and practically irrelevant) at the time of mechanical experiments, it was finally stressed during 19th and 20th centuries when Physics recognized to be working in terms of fields.

With special relativity (SR) Einstein realized that the physical properties of the electromagnetic field are fundamentally entangled with our notion of space and time (or, more correctly, spacetime) on which our observational protocols rely, for example through synchronization. SR is in fact a good model of spacetime and electromagnetic field in which observational protocols are made explicit. As such, and in view of later studies of general relativity (GR), SR is fundamentally a theory of empty space, with electromagnetic field but no other matter.

In $f(R)$-theories the electromagnetic field is described equivalently by $g$ and $\tilde{g}$ (as well as by any conformal representative). Accordingly, no purely electromagnetic measure can observe any difference between any conformal gauge fixing. Also no purely electromagnetic measure can fix a scale of our observations. In the Dirac notations scales are not gauge invariant and hence are not observable at fundamental level. Thus one could ask what we are measuring in astrophysics and cosmology? 
This issue is well known (see [12]). Also, in GR there is no non-trivial generally invariant observable or at least no such quantity is known. In GR also the value of scalars at a fixed point is not gauge invariant (the Lie derivative of a scalar at a point does not vanish in general). This is essentially the starting point of the celebrated hole argument in GR which shows that there is no physical meaning in fixing a point in spacetime. Rovelli proposed that matter basically breaks the gauge invariance and allows to define observable which are observed against the matter background; see [12]. This is philosophically (if not technically) quite close to the EPS approach; it is matter and light which allows to infer a description of gravitational field. It is matter (and masses) which allows to break the conformal gauge. For example, in order to know the mass of the sun one has to use (in the classical limit) the Kepler laws. It is a measure of orbit size $r$ and orbit period $T$ which allows to measure the mass of the sun

$$
\frac{4 \pi^{2}}{M G}=\frac{T^{2}}{r^{3}} \quad \Rightarrow M G=\frac{4 \pi^{2} r^{3}}{T^{2}}
$$

Of course none of this is conformally invariant; neither the size of the orbit nor the orbit period. Accordingly, the mass $M G$ does scale non trivially under conformal transformations. If one defines the unit for masses so that $G=1$ as it is often done then the mass $M$ itself is not a conformally invariant quantity. On the other hand the mass is the source of gravitational field and as such it must be somehow observable. And there are other hints that support the fact that traditional observation in astrophysics and cosmology are in fact held in a specific conformal gauge fixing. The traditional astronomical observational protocols do in fact define a space distance (which depends on the conformal gauge fixing). Also in standard GR, asymptotics of the metric does in fact freeze the conformal degrees of freedom of the metric.

Hereafter we shall discuss the conformal origin of the mass in a Weyl universe. We shall review some models in which we showed that one can take advantage of the freedom in Weyl geometries to describe a fluid on an arbitrary (e.g. flat) metric. In these models the gravitational field is described by the connection (or equivalently by the covector $A$ ) while the metric structure $g$ describes the metric protocols (distances and clocks) and the electromagnetic field, which is in fact conformally invariant. We shall also show that conservation laws of the fluid (which are in the end the foundation of mass) do in fact break conformal invariance and one obtains the standard relations obtained in standard GR, though in this more general setting, provided that the conformal gauge is fixed accordingly; see [13] and [14].

This toy model also shows how one could describe masses in some sense without curvature (of course masses do produce gravitational field which is encoded in some curvature, namely the curvature of the connection which is encoded by $A_{\varepsilon}$ ) but in a (possibly flat) metric background which is fairly decoupled by the gravitational phenomenology. The metric (i.e. a given gauge fixing of the conformal gauge) is in fact endowed with a physical meaning once one defines distances and clocks (which also breaks the conformal gauge).

They also shows that Einstein had other possibilities to extend SR to describe masses which unfortunately where out of reach for the geometrical technology of his time since a theory for general connections was still on the way. When Einstein was led to consider the gravitational field he was forced to take the only direction that his contemporary geometric technologies allowed to use. Gravitation was identified with the curvature of a spacetime metric, leading to standard GR. 
At that time, general connections were still on the way of being invented and the only curvature known was metric curvature.

When general connections were finally invented (maybe feeling something to be improved in GR in the relation among electromagnetic field, gravitational field and matter) Einstein tried the way of a unifying theory of electromagnetic and gravitational fields in which both fields were the output of a connection. Einstein could not reach a satisfactory solution to this problem (later solved in [15]).

\section{Mathematical vs. Physical equivalence}

EPS clearly points towards a framework a là Palatini in which dynamics enforces EPS compatibility of metric and connection. The metric $g$ and the connection $\Gamma$ are, by construction, related to lightcones and free fall, respectively. We shall assume also that $g$ is the metric related to distances and clocks (also in view of the fact that lightcones determine the conformal class $[g]$, not a representative $g$ ).

Palatini $f(R)$ gravity has been often considered trivial in view of equivalence to scalar-tensor gravity which is well understood. In some sense, since the connection is metric, it is described by a metric $\tilde{g}$ which being conformally equivalent to $g$ describes lightcones on equal footing of $g$. Then $\tilde{g}$ describes both lightcones and free fall. One metric (namely, $\tilde{g}$ ) describes both structures as it is assumed in standard GR.

Before discussing how matter can fix the conformal gauge, let us discuss in a simple mechanical example how mathematical equivalence can or cannot be extended to a full physical equivalence. Here the point is that even in view of a mathematical equivalence which in principle allows to map one description into the other, observational protocols may break the equivalence. This may happen especially when observations are difficult and one is unable to measure everything but just some quantities can be measured more or less directly, as it happens in astrophysics and cosmology.

Imagine we are given a material point constrained on a straight line free to move under unknown forces to be studied. We know that one can measure the position $x$ and the the momentum $\pi$. We can easily plot $x$ and $\pi$ in their time evolution.

One question which is easily asked is whether the system can be described as a Hamiltonian system. For it, one should identify two quantities $q$ and $p$, give a Hamiltonian function $H(q, p)$ and relate the evolution of the system and the solutions of the Hamilton equations

$$
\begin{aligned}
& \dot{q}=\frac{\partial H}{\partial p} \\
& \dot{p}=-\frac{\partial H}{\partial q}
\end{aligned}
$$

By observing the motion of the point in the range (a) $t=10 . .20$ one could make an educated guess for a harmonic oscillator. By a closer look one could fit the data very well by a function

$$
x=A \cos \left(\omega\left(t+t_{0}\right)\right)+\lambda
$$

with $A=10, \omega=3, \lambda=1, t_{0}=0$. 

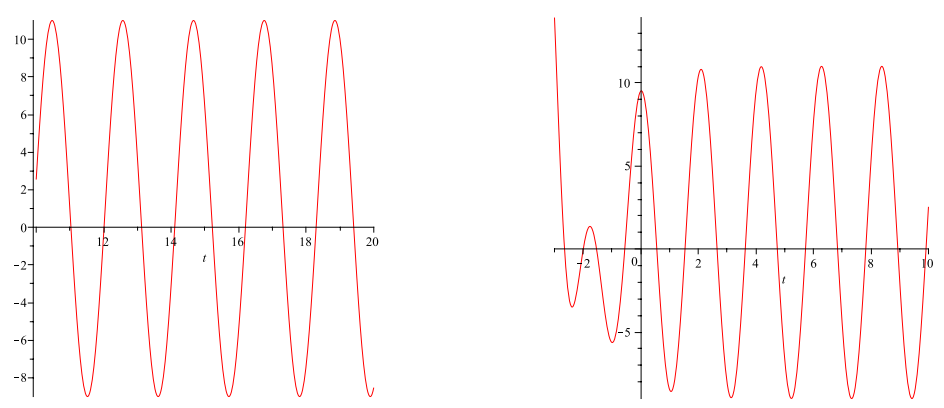

Figure 1: $x(t)$ in the time range a) $t=10 . .20$.

b) $t=-3 . .10$.

The system is thence described by the Hamiltonian

$$
\bar{H}=\frac{1}{2} p^{2}+\frac{\omega^{2}}{2} q^{2}-\lambda \omega^{2} q
$$

Accordingly, one can guess the force to be a harmonic force plus a constant force.

After that one obtains a bigger dataset $(b)$ which includes the time range $t=-3 . .10$. The new dataset clearly disagrees with previous guesses. It seems that something awkward happened at time $t=0$. One could either suppose some extra temporary force acted and it is then switched off, or look for as single time dependent force explaining both datasets.

In fact a very good fit can be obtained by choosing a Hamiltonian

$$
H=\frac{\alpha^{2}}{2} p^{2}+\frac{\omega^{2}}{2 \alpha^{2}} q^{2}-\lambda \frac{\omega^{2}}{\alpha} q+\frac{\dot{\alpha}}{\alpha} p q
$$

for a parameter function $\alpha(t)=1-e^{-(t+2)}$. Of course this model is a big step forward since it perfectly describes the whole dataset.

Then one can try to look for a canonical transformation to simplify the model. In particular one can define new (one parameter family of) canonical coordinates

$$
\begin{aligned}
& Q=\frac{q}{\alpha(t)}-\lambda \\
& P=\alpha(t) p
\end{aligned}
$$

and check that the new Hamiltonian is simply

$$
K=\frac{1}{2} P^{2}+\frac{\omega^{2}}{2} Q^{2}
$$

Obviously, the canonical transformation establishes a very well founded mathematical equivalence between the two Hamiltonian systems describes by $H$ and $K$ (which is in fact one Hamiltonian system with two different local representations).

To what extent though the system is a pure harmonic oscillator on the physical stance? To answer the question maybe it is worth considering some remarks. First, the dynamics $K$ is much simpler than the dynamics of $H$. Second, in the $K$ framework there is an observable $x=\alpha(t)(Q+\lambda)$ which fits the dataset as perfectly as $q$ does in the $H$ framework. Third, in the $H$ framework the observable used for fitting is simply $x=q$. 
Accordingly, we have two mathematically equivalent frameworks, one in which the dynamics is particularly simple, the other in which what we observe (i.e. our observational protocols) is particularly simple. Because of the particular situation either we find a way of observing directly the quantity $Q$ (which would make the $K$ framework superior under all viewpoints) or we have to resign to have two frameworks each simpler under a different viewpoint.

Let us also finally remark that the transformation between the two frameworks, namely $q=$ $\alpha(t)(Q+\lambda)$, can be directly related to a (time-dependent) mismatch of the protocol of measuring the position. It is as if, besides changing the origin $\lambda$ of the position reference frame, we did change the unit of distances by a (time-dependent) factor $\alpha(t)$ (imagine for example we are using ultrasounds to define distances and the speed of sound in changing with time (for example due to a change in pressure); or imagine we are using a conformal metric and the conformal factor is depending on the matter density which is changing in a ever expanding universe). The mismatch does not emerge in datasets covering intervals in which the function $\alpha$ can be considered constant (as it happens in the dataset $(a)$ ) while it becomes manifest once the dependence of $\alpha$ on time can be appreciated.

Though we do not need to recall that in cosmology the protocols for defining and measuring distances are quite obscure from a fundamental perspective and depend on many aspects of the underlying model and many physical assumptions, we are not here claiming that a similar mechanism can explain cosmological observations. However, one should understand in details how this is not the case.

This rather trivial example in any event shows how one should not use mathematical equivalence to dismiss a framework without carefully review the observational protocols and verifying their compatibility with the equivalence transformations, especially in cosmology where we know from the very beginning that most of the time we are measuring quantities that are not gauge covariant.

\section{Fluids in a Weyl Geometry}

In stadard GR spacetime geometry is a Lorentzian structure $(M, g)$. The metric $g$ determines both lightcones and free fall of massive particles. While null geodesics are conformally invariant, timelike geodesics are not. A conformal metric $\tilde{g}$ has the same null geodesics (possibly differently parametrized but in any event the same worldlines) though different timelike geodesics worldlines. Accordingly on a given metric background $(M, g)$ not any timelike congruence of trajectories is geodesic and one has strong constraints on possible motions of fluids' particles.

Of course it is weird to regard as constraints the conditions which makes the motion of the fluids' particles determined by the metric structure (which in standard GR is the gravitational field). However, in the wider framework of Weyl geometries the metric structure is a gauge fixing of the conformal gauge freedom and the gravitational field is entirely encoded in the independent connection (or equivalently the covector $A$ ). It would be equally weird in this context to select the allowed fluids depeding on a gauge fixing. To be more precise this would make observable (through the observations of fluids) a gauge fixing.

Thus it is precisely Dirac theory of observability which indicates that one should be able to describing a fluid by any timelike congruences of curves. Then the gravitational field is described 
by the choice of a covector $A_{\varepsilon}$ and this selects a class of possible fluid motions. Also the other way around; observing the motion of particles of a fluid one should be able to spot which gravitational field influences the fluid, making the gravitational field clearly observable through its effects on matter.

Let us hereafter briefly review the results of [13]. Let us consider, on a spacetime $M$ of dimension $m$, a conformal structure $(M, \mathfrak{C})$. The conformal structure is a class of Lorentzian metrics

$$
\mathfrak{C} \equiv[g]=\left\{\tilde{g}=\Phi^{2} \cdot g\right\}
$$

Let us remark that the notion of tikelike (null or spacelike, respectively) does not depend on the representative of the conformal class. Then we can consider a $\mathfrak{C}$-timelike vector field $u$. The integral curves of $u$ are $\mathfrak{C}$-timelike trajectories by construction. On the contrary, one can start from any $\mathfrak{C}$-timelike congruence of worldlines and define the infinitesimal generator $u$ of the family. For any gauge fixing of the conformal structure $g \in \mathfrak{C} \equiv[g]$ one can normalize $u$ to be a $g$-unit vector. Let us denote the normalized vector by $n$.

Let $\gamma: \mathbb{R} \rightarrow M$ be an integral curve of the vector field $n$. We can arbitrarily reparametrize the curve $\gamma$ to obtain a different representative $\gamma \circ \phi$ of the same trajectory, the change of parameter being described by the diffeomorphism $\phi: \mathbb{R} \rightarrow \mathbb{R}$. If the original curve $\gamma$ was a $\Gamma$-geodesic motion (for a connection $\Gamma$ ) then $\gamma \circ \phi$ is a $\Gamma$-geodesic trajectory. Accordingly, one has

$$
n^{\mu} \nabla_{\mu}^{(\Gamma)} n^{\alpha}=\varphi \cdot n^{\alpha}
$$

for some scalar field $\varphi(x)$.

In standard GR one has no much choice for the fluid flow lines generated by $n$; the connection is freezed to be the Levi-Civita connection of $g$ and the vector field $n$ has to be selected in the small class of geodesic fields. In a Weyl setting one has a wider freedom in chosing the connection in the class of EPS-compatible connections given by (1.1). One can rely on this freedom to show that for any timelike vector field $n$ there exists an EPS-compatible connection $\Gamma$ for which $n$ is $\Gamma$-geodesic, i.e. (3.1) holds true. One can easily check that $A$ has to fixed as

$$
A_{v}=n^{\mu} \nabla_{\mu}^{(g)} n_{v}+\varphi n_{v}
$$

Notice how, once $u$ is given and a parametrization of curves is fixed by choosing the scalar field $\varphi$, one can uniquely determine the covector $A$ and thence the connection $\Gamma$. If one started from a different conformal representative $\tilde{g}=\Phi^{2} \cdot g$ this would amount to redefine accordingly the covector $\tilde{A}=A+d \ln \Phi$ to obtain the same $\Gamma$, the unit vector $\tilde{n}^{\lambda}=\Phi^{-1} n^{\lambda}$ and the scalar field $\tilde{\varphi}=\Phi^{-1}\left(\varphi-n^{\mu} \nabla_{\mu}^{(*)} \ln \Phi\right)$, where $\nabla_{\mu}^{(*)}$ denotes the covariant derivative in the case it is independent of the connection. We refer to [13] for details and proofs.

\section{Fluid Conservation Laws}

The fluid is described, besides by its flow lines which are generated by $u$, by two scalar fields $\rho(x)$ and $p(x)$ describing particle density and pressure. The (symmetric) energy-momentum tensor of the fluid is in the form

$$
T_{\mu \nu}=p g_{\mu \nu}+(p+\rho) n_{\mu} n_{\nu}
$$


In standard GR, one has a strict relation between Bianchi identities (associated to field equations), conservation of energy-monentum tensor $\nabla_{v} T^{\mu v}=0$, matter field equations (which together with fluid equation of state determine the evolution of the fluid) and conservation laws which are associated to conservation of the number of particles (and the fluid energy).

In Weyl setting one should describe the same sort of relations, by suitably specifying when covariant derivatives are induced by $g$ and when they are induced by $\Gamma$.

The conservation of energy-momentum tensor is

$$
\begin{gathered}
\nabla_{v}^{(\Gamma)} T^{\mu v}=0 \quad \Rightarrow \\
(p+\rho) \nabla_{v}^{(\Gamma)} n^{v}=(p-\rho) \varphi-n^{v} \nabla_{v}^{(*)} \rho \\
\left(g^{\mu v}+n^{\mu} n^{v}\right) \nabla_{v}^{(*)} p=2 p n^{v} \nabla_{v}^{(g)} n^{\mu}
\end{gathered}
$$

where we used (1.1) and (3.2).

By starting from a different conformal gauge fixing $\tilde{g}=\Phi^{2} \cdot g$ one has different pressure and density, since physical rods and therefore measures depend on the choice of the conformal factor $\Phi$. In principle one sets $\tilde{p}=\Phi^{n} p$ and $\tilde{\rho}=\Phi^{n} \rho$ (with the power $n$ to be determined later in view of conservation of the number of particles) and the energy momentum tensor is

$$
\tilde{T}_{\mu v}:=\tilde{p} \tilde{g}_{\mu \nu}+(\tilde{p}+\tilde{\rho}) \tilde{n}_{\mu} \tilde{n}_{\nu}=\Phi^{n+2}\left(p g_{\mu \nu}+(p+\rho) n_{\mu} n_{v}\right)=\Phi^{n+2} T_{\mu \nu}
$$

Of course, unless $n=-2$ (as we shall see below this happens in dimension $m=3$ ), the energymomentum tensor is not conformally invariant and, more importantly, its conservation is not preserved by conformal transformations. If $T_{\mu \nu}$ is conserved then in general $\tilde{T}_{\mu \nu}$ is not. Accordingly, one has to specify in which gauge the conservation of energy-momentum tensor has to be imposed. We shall discuss this issue below in greater detail.

Also conservation of number of particles can be discussed at kinematical level. If $\rho$ is related to the density of particles of the fluid, then one would like to define a quantity $J^{\mu}$ which can be integrated on a spatial region $\Sigma$ to determine the number $N_{\Sigma}$ of fluid particles hitting $\Sigma$. The number $N_{\Sigma}$ must be constant along the flow of $n$.

An object to be integrated along an hypersurface $\Sigma$ is a $(m-1)$-form. There is a natural choice:

$$
J=\sqrt{g} T^{\mu v} n_{v} d s_{\mu}=: J^{\mu} d s_{\mu} \quad N_{\Sigma}:=\int_{\Sigma} J^{\mu} d s_{\mu}
$$

The number of particles $N_{\Sigma}$ is conserved along the flow of $n$ iff one has $d J=0$. The current $J$ is sensible to conformal transformations since measures are. It can be redefined out of any conformal framework and one has

$$
\tilde{J}=\sqrt{\tilde{g}} \tilde{T}^{\mu v} \tilde{n}_{v} d s_{\mu}=\Phi^{m} \sqrt{g} \Phi^{n-2} T^{\mu v} \Phi n_{v} d s_{\mu}=\Phi^{m+n-1} J
$$

The integral $N_{\Sigma}$ counts how many fluid particles hit the region $\Sigma$ and as such Physics imposes that it has to be independent of the conformal framework. This forces $n$ to be fixed as $n=1-m$ so that $J$ is conformally invariant and its integral is accordingly invariant. 
This thence prescribes the following conformal transformations

$$
\tilde{p}=\Phi^{1-m} p \quad \tilde{\rho}=\Phi^{1-m} \rho
$$

This could be expected since $\rho$ and $p$ denote the spatial densities (of particles and pressure).

\section{Conservation of $T_{\mu v}$}

In a general Weyl context the conservation of energy-momentum tensor and its relation with conservation of the current $J$ needs to be deeply reviewed. One has in fact from conservation of number of particles:

$$
\partial_{\mu} J^{\mu}=\nabla_{\mu}^{(\Gamma)}\left(\sqrt{g} T^{\mu v} n_{v}\right)=\sqrt{g}\left(\nabla_{\mu}^{(\Gamma)} T^{\mu v} n_{v}+T^{\mu v} \nabla_{\mu}^{(\Gamma)} n_{v}\right)+\nabla_{\mu}^{(\Gamma)} \sqrt{g} T^{\mu v} n_{v}=0
$$

In standard GR one has $\Gamma=\{g\}$ and hence $\nabla_{\mu} \sqrt{g}=0$; if $n$ is a Killing vector also $T^{\mu \nu} \nabla_{\mu} n_{v}=0$ and conservation of particles, $d J=0$, is implied by conservation of the energy-momentum stress tensor (which, usually, is eventually implied by Bianchi identities). However, we have to observe that conservation of $J$ does not on the contrary imply conservation of energy-momentum tensor. Only the projection of conservation laws along $n$ is in fact used. Moreover, being $n$ a Killing vector is a sufficient but by no means necessary condition.

In a general Weyl context one has in fact

$$
\partial_{\mu} J^{\mu}=\sqrt{g}\left(\nabla_{\mu}^{(\Gamma)} T^{\mu v} n_{v}+T^{\mu v} \nabla_{\mu}^{(\Gamma)} n_{v}\right)+m \sqrt{g} A_{\mu} T^{\mu v} n_{v}=0
$$

Thence one should somehow impose that in general one has

$$
T^{\mu v} \nabla_{\mu}^{(\Gamma)} n_{v}=0 \quad T^{\mu v} A_{\mu} n_{v}=0
$$

without being too demanding on the vector $n$.

Here the situation is easier for dust $(p=0)$. Accordingly, let us first consider this case.

$$
\begin{aligned}
T^{\mu v} \nabla_{\mu}^{(\Gamma)} n_{v} & =\rho n^{\mu} n^{v} \nabla_{\mu}^{(\Gamma)}\left(n^{\alpha} g_{\alpha v}\right)= \\
& =\rho\left(n_{\alpha} n^{\mu} \nabla_{\mu}^{(\Gamma)} n^{\alpha}-2 n^{\mu} A_{\mu}\right)=\rho(-\varphi+2 \varphi)=\varphi \rho
\end{aligned}
$$

Now we have to stress that the scalar $\varphi$ can be chosen at will being related to the choice of a parametrization of worldlines. Here of course we would like to fix $\varphi=0$. This corresponds to require that the integral curves of $n$ are not only geodesics trajectories of $\Gamma$, but in fact they are geodesic motions. We here started to be general enough but we are forced back to geodesic motions.

As far as the second condition is concerned one has

$$
T^{\mu v} A_{\mu} n_{v}=-\rho A_{\mu} n^{\mu}=\rho \varphi=0
$$

which is also satisfied for $\varphi=0$.

The situation with pressure is more complicated.

One can easily show that

$$
T^{\mu v} \nabla_{\mu}^{(\Gamma)} n_{v}=p \nabla_{\mu}^{(g)} n^{\mu}+(2 \rho-m p) \varphi \quad T^{\mu v} A_{\mu} n_{v}=\rho \varphi
$$


In fact one has:

$$
\begin{aligned}
T^{\mu v} \nabla_{\mu}^{(\Gamma)} n_{v} & =p g^{\mu v} \nabla_{\mu}^{(\Gamma)} n_{v}+(p+\rho) n^{v} n^{\mu} \nabla_{\mu}^{(\Gamma)} n_{v}= \\
& =p \nabla_{\mu}^{(\Gamma)} n^{\mu}+2 p n^{\mu} A_{\mu}+(p+\rho) n_{\alpha} n^{\mu} \nabla_{\mu}^{(\Gamma)} n^{\alpha}-2(p+\rho) n^{\mu} A_{\mu}= \\
& =p \nabla_{\mu}^{(\Gamma)} n^{\mu}-2 p \varphi-(p+\rho) \varphi+2(p+\rho) \varphi=p \nabla_{\mu}^{(\Gamma)} n^{\mu}+2 \rho \varphi= \\
& =p \nabla_{\mu}^{(g)} n^{\mu}+p n^{\lambda}\left(g^{\mu \varepsilon} g_{\lambda \mu}-\delta_{\lambda}^{\mu} \delta_{\mu}^{\varepsilon}-\delta_{\mu}^{\mu} \delta_{\lambda}^{\varepsilon}\right) A_{\varepsilon}+2 \rho \varphi= \\
& =p \nabla_{\mu}^{(g)} n^{\mu}+p n^{\lambda}\left(\delta_{\lambda}^{\varepsilon}-\delta_{\lambda}^{\varepsilon}-m \delta_{\lambda}^{\varepsilon}\right) A_{\varepsilon}+2 \rho \varphi=p \nabla_{\mu}^{(g)} n^{\mu}+(2 \rho-m p) \varphi
\end{aligned}
$$

and one has to face the incompressibility condition $\nabla_{\mu}^{(g)} n^{\mu}=0$. This can be solved by using the freedom in the conformal gauge fixing. One can show that there is always a conformal representative for which

$$
\nabla_{\mu}^{(\tilde{g})} \tilde{n}^{\mu}=0
$$

and then in this conformal frame we fix $\tilde{\varphi}=0$.

One has in fact

$$
\begin{aligned}
\nabla_{\mu}^{(\tilde{g})} \tilde{n}^{\mu} & =\nabla_{\mu}^{(g)} \tilde{n}^{\mu}-\tilde{n}^{\lambda}\left(g^{\mu \varepsilon} g_{\lambda \mu}-\delta_{\lambda}^{\mu} \delta_{\mu}^{\varepsilon}-\delta_{\mu}^{\mu} \delta_{\lambda}^{\varepsilon}\right) \partial_{\varepsilon} \ln \Phi= \\
& =\nabla_{\mu}^{(g)} \tilde{n}^{\mu}-\tilde{n}^{\lambda}\left(\delta_{\lambda}^{\varepsilon}-\delta_{\lambda}^{\varepsilon}-m \delta_{\lambda}^{\varepsilon}\right) \partial_{\varepsilon} \ln \Phi=\nabla_{\mu}^{(g)} \tilde{n}^{\mu}+m \tilde{n}^{\varepsilon} \partial_{\varepsilon} \ln \Phi= \\
& =\frac{1}{\Phi}\left(\nabla_{\mu}^{(g)} n^{\mu}+(m-1) n^{\varepsilon} \partial_{\varepsilon} \ln \Phi\right)
\end{aligned}
$$

Now, as a matter of fact whaterver $\nabla_{\mu}^{(g)} n^{\mu}$ is, one can always find a conformal factor $\Phi$ such that

$$
n^{\varepsilon} \partial_{\varepsilon} \ln \Phi=-\frac{1}{m-1} \nabla_{\mu}^{(g)} n^{\mu}
$$

(fix coordinates in which $n=\partial_{0}$ ). Using such a conformal factor to change conformal representative, in the new conformal frame one has $\nabla_{\mu}^{(\tilde{g})} \tilde{n}^{\mu}=0$.

For the second condition to hold one has

$$
T^{\mu v} A_{\mu} n_{v}=-(\rho+p) A_{\mu} n^{\mu}+p A_{\mu} n^{\mu}=-\rho A_{\mu} n^{\mu}=\rho \varphi
$$

which also vanishes under the same condition.

Thus in general one needs not to require that $n$ is Killing. In fact the fluid (with pressure) selects a preferred conformal frame $\tilde{g}$. In that preferred frame one has a preferred Weyl connection with

$$
\tilde{A}_{v}=\tilde{n}^{\mu} \nabla_{\mu}^{(\tilde{g})} \tilde{n}_{v}
$$

for which $\tilde{n}^{\mu} \nabla_{\mu}^{(\Gamma)} \tilde{n}^{v}=0$. With these choices, not only the fluid velocities are a geodesic field, but the conservation law $d J=0$ is equivalent to the conservation of fluid energy-momentun tensor, $\nabla_{\mu}^{(\Gamma)} T^{\mu v}=0$.

\section{Conformal transformations}

If we have to take seriously Weyl geometry and EPS framework, once matter is added then it must be free falling along geodesics of $\Gamma$, not along geodesics of $\{g\}$ as in standard GR.

Now it is well known that massive particles move along the eikonal approximation of the corresponding field (e.g. Klein-Gordon field). Thus in turn the EPS request is in fact a prescription to 
fix matter-gravity coupling so that it finally results that massive particles do move along geodesics of $\Gamma$.

This has longly been discussed in literature (see [6]), whether minimal coupling should be imposed in the Einstein frame or in the Jordan frame, i.e. whether matter coupled naturally to the metric $g$ or to the connection $\Gamma$.

Let us here consider the special case of Klein-Gordon matter field. We shall show that KleinGordon equations are almost covariant with respect to conformal transformation (which in $f(R)$ models connect $g$ to the connection $\Gamma$ which is in fact the Levi-Civita connection to a conformal metric $\tilde{g}$ ).

In fact, a conformal transformation maps a Klein-Gordon equation into a similar equation with a point-dependent mass (actually a function of the conformal factor). Thus in most situations (i.e. in regions where the conformal factor is almost constant as in virtually all cases regarding tests of GR) the conformal transformations finally amounts to a rescaling of effective masses. When the conformal factor is near 1 (as in standard GR or in the Solar system) then the conformal transformation leaves Klein-Gordon equation approximately invariant and the rescaling can be hidden by observation errors.

Still when gravity-matter interactions are important (as in non-vacuum solutions, e.g. FriedmanRobertson-Walker cosmological solutions) or when the conformal factor is non-trivial (e.g. in strong coupling regime) then the mass rescaling can be important.

Let us fix dimension $m=4$. The transformation rules of the Klein-Gordon equation with respect to conformal rescaling of the metric and the scalar field can be easily obtained using a simple fact concerning the conformal Laplacian operator $\square=\square-\frac{1}{6} R$.

One can easily see that in view of the transformation $g \mapsto \tilde{g}=\Phi g, \tilde{\varphi}=\Phi^{n} \varphi$ (for $n=-\frac{1}{2}$ ) one has:

$$
\sqcup \varphi+\mu^{2} \varphi=\Phi^{\frac{3}{2}} \tilde{\square} \tilde{\varphi}+\mu^{2} \Phi^{\frac{1}{2}} \tilde{\varphi}=\Phi^{\frac{3}{2}}\left(\tilde{\square} \tilde{\varphi}+\frac{\mu^{2}}{\Phi} \tilde{\varphi}\right)=0
$$

If one starts from a Minkowski spacetime, then $R=0$ and $\square=\square$. In this case, a conformal spacetime $\tilde{g}_{\mu \nu}=\Phi \cdot \eta_{\mu \nu}$ is conformally flat and one has the scalar field to obey the equation

$$
\tilde{\square} \tilde{\varphi}-\frac{1}{6} \tilde{R} \tilde{\varphi}+\frac{\mu^{2}}{\Phi} \tilde{\varphi}=\tilde{\square} \tilde{\varphi}+\left(\frac{\mu^{2}}{\Phi}-\frac{1}{6} \tilde{R}\right) \tilde{\varphi}=0
$$

This is again a Klein-Gordon-like equation with an effective mass

$$
\tilde{\mu}^{2}=\frac{\mu^{2}}{\Phi}-\frac{1}{6} \tilde{R}
$$

Let us remark that even starting from a massless Klein-Gordon field $(\mu=0)$ the final effective mass receives a contribution from the curvature of the conformal metric. Second, the curvature itself can be expressed in terms of the conformal factor (since the original metric is flat) and one has

$$
\tilde{\mu}^{2}=\frac{\mu^{2}}{\Phi}-\frac{1}{6 \Phi}\left(-3 \square \ln \Phi-\frac{3}{2 \Phi} \tilde{\nabla}_{\mu} \Phi \tilde{\nabla}^{\mu} \Phi\right)=\frac{\mu^{2}}{\Phi}+\frac{1}{2 \Phi^{2}} \square \Phi-\frac{1}{4 \Phi^{2}} \tilde{\nabla}_{\mu} \Phi \tilde{\nabla}^{\mu} \Phi
$$

As a side effect, a solution of Klein-Gordon coupled with a metric $g$ is a solution of a KleinGordon (with an effective mass depending on the conformal mass) for any conformal metric $\tilde{g}$. This 
is a first evidence that one can eventually have matter fields coupled with the Einstein metric and hence in eikonal approximation, test particles free falling along geodesics of $\tilde{g}$. All these effects are completely trivial in vacuum models when $\Phi=$ const, though they are switched on within matter.

\section{Klein-Gordon Lagrangian}

It is also interesting the transformation laws for Klein-Gordon fields at the Lagrangian level. Let us consider a spacetime of dimension $m$ in which a Klein Gordon field $\varphi$ is described by the matter Lagrangian

$$
L_{K G}=\frac{\sqrt{g}}{2}\left(\nabla_{\mu}^{(*)} \varphi \nabla^{(*) \mu} \varphi-\mu^{2} \varphi^{2}\right)
$$

where $\nabla_{\mu}^{(*)}$ denotes the covariant derivative when it is independent of a connection and it reduces to the ordinary partial derivative.

The conformal transformation acts on the metric

$$
\tilde{g}_{\mu v}=\Phi \cdot g_{\mu v} \quad \Rightarrow \quad \sqrt{\tilde{g}}=\Phi^{\frac{m}{2}} \sqrt{g} \quad g^{\mu v}=\Phi \cdot \tilde{g}^{\mu v}
$$

and on the Klein-Gordon field

$$
\tilde{\varphi}=\Phi^{n} \varphi
$$

with the exponent to be later determined so that the transformed field $\tilde{\varphi}$ is still described by a Klein-Gordon-like Lagrangian.

By few simple manipulations one can show that fixing $n=\frac{1}{2}-\frac{m}{4}$ one has

$$
L_{K G}=\frac{\sqrt{\tilde{g}}}{2}\left(\nabla_{\mu}^{(*)} \tilde{\varphi} \nabla^{(*) \mu} \tilde{\varphi}-\tilde{\mu}^{2} \tilde{\varphi}^{2}\right)+\nabla_{\mu}^{(*)} f^{\mu}
$$

where we set

$$
\tilde{\mu}^{2}=\frac{\mu^{2}}{\Phi}+\frac{(m-6)(m-2)}{16 \Phi^{2}} \tilde{\nabla}_{\mu} \Phi \tilde{\nabla}^{\mu} \Phi+\frac{m-2}{4 \Phi^{2}} \square \Phi
$$

and

$$
f^{\mu}=-\frac{\sqrt{\tilde{g}}}{2 \Phi}\left(\frac{1}{2}-\frac{m}{4}\right) \nabla^{(*) \mu} \Phi \tilde{\varphi}^{2}
$$

The mass $\tilde{\mu}^{2}$ in dimension $m=4$ reduces to (6.2) while the divergence term does not contribute to matter field equations.

Klein-Gordon matter then is not preserved by conformal transformations. A Klein-Gordon field in one gauge is not strictly Klein-Gordon in a conformal gauge. Accordingly, if the dynamics is imposed to be minimally coupled in one framework it is not in the other. It is though approximately Klein-Gordon, for example in regions where $\tilde{\mu}^{2}$ is approximately constant. Also in these cases the value of the actual mass $\mu$ is modified to a different effective mass $\tilde{\mu}$.

\section{Conclusions and Perspectives}

We showed that for any conformal structure $[g]$ on a spacetime $M$ and for any timelike vector field $u$ one can always determine an EPS-compatible connection $\Gamma$ for which the vector field $u$ is geodesic. Then one can determine the conformal frame and parametrizations along worldlines so 
that one has the standard relation among the different conservations associated to the fluid. We also determined how matter Lagrangian and field equations behave under conformal transformations.

Of course we are not here suggesting that the model we presented here is physically sound. One should specify a dynamics and then investigate the relation with Bianchi identities. We chose this model to show that had Einstein known it, he could have possibly tried this way to model matter and gravity and make them compatible with SR.

This research is part of a larger project aiming to model a generic self-gravitating fluid in EPS formalism. One can specify the conformal structure to be the Minkowskian one by setting $g=\eta$ and still be free to model any congruence of (timelike) worldlines as the flow of a fluid. In this framework the gravitational field is encoded into the covector $A$ which in turn determines the Weyl connection $\Gamma$.

More investigations are required in this direction to show that in fact one can set the coupling with matter so that the dynamics in fact realized what prescribed in EPS (i.e. free falling determined by $\Gamma$ ). We here first sketched this at the level of field equations and Lagrangian for a Klein-Gordon field while it should be worked out more generally.

Moreover, all observational protocols commonly used in GR rely on uniqueness of parallel transport. When conformal transformations are considered one has automatically a whole family of parallel transports. What impact have these on observational protocols (and then on GR tests) are to be discussed in details as well.

Let us stress that in the EPS setting there is no freedom in choosing the connection associated to free fall. In EPS framework the free fall of particles is by construction described by the connection $\Gamma$ while the Levi-Civita connection of $g$ plays just the kinematical role of reference frame in the affine space of connections (which is moreover conformally covariant since one can start from any representative of the conformal structure).

The extra degrees of freedom to determine in $\Gamma$ are thence encoded into the covector $A$ which is kinematically free to be generic. The dynamics of the theory determines then the connection $\Gamma$ fixing the covector $A$ in terms of matter fields and $g$.

Of course Weyl geometries are affected by physical interpretation problems mainly related to the (possibly non-trivial) holonomy of the connection $\Gamma$; see [8]. However, these problems arise only if $\Gamma$ is not metric while metric connections do not generate any physical problem of this kind and have just to be interpreted correctly. We stress that in all $f(R)$ models with non-exotic matter (or in vacuum) dynamics forces a posteriori the connection $\Gamma$ to be automatically metric (and in fact to be the Levi-Civita connection of a metric conformal to the $g$ originally entering the Lagrangian; see [9] and [10]), unless one introduces a matter Lagrangian in which matter couples directly with the connection $\Gamma$ (a case in which the connection can be also not metric; see [7]).

\section{Acknowledgments}

This work is supported by INFN (Iniziativa Specifica NA12), by the local research project 2011 of Dipartimento di Matematica of University of Torino (Italy) and by INdAM-GNFM. 


\section{References}

[1] S. Capozziello, M. De Laurentis, V. Faraoni A birdÕs eye view of $f(R)$-gravity (2009); arXiv:0909.4672

[2] T.P. Sotiriou, S. Liberati, Metric-affine $f(R)$ theories of gravity, Annals Phys. 322 (2007) 935-966; gr-qc/0604006

[3] S. Nojiri, S.D. Odintsov, Modified gravity as realistic candidate for dark energy, inflation and dark matter, AIP 9 Conf.Proc. 1115, 2009, 212-217; arXiv:0810.1557

[4] M. Di Mauro, L. Fatibene, M. Ferraris, M. Francaviglia, Further Extended Theories of Gravitation: Part I Int.J.Geom.Meth.Mod.Phys.7:887-898,2010; arXiv:0911.2841v2 [gr-qc]

[5] L. Fatibene, M. Ferraris, M. Francaviglia, S. Mercadante Further Extended Theories of Gravitation: Part II Int.J.Geom.Meth.Mod.Phys.7:899-906,2010; arXiv:0911.2842v2 [gr-qc]

[6] G. Magnano, L.M. Sokolowski, On Physical Equivalence between Nonlinear Gravity Theories Phys.Rev. D50 (1994) 5039-5059; gr-qc/9312008

[7] L.Fatibene, M.Francaviglia, S. Mercadante, Matter Lagrangians Coupled with Connections Int. J. Geom. Methods Mod. Phys. Volume: 7, Issue: 5 (2010), 1185-1189; arXiv: 0911.2981

[8] J.Ehlers, F.A.E.Pirani, A.Schild, The Geometry of Free Fall and Light Propagation, in General Relativity, ed. L.OÕRaifeartaigh (Clarendon, Oxford, 1972).

[9] M.Di Mauro, L. Fatibene, M.Ferraris, M.Francaviglia, Further Extended Theories of Gravitation: Part I , Int. J. Geom. Methods Mod. Phys. Volume: 7, Issue: 5 (2010), pp. 887-898; gr-qc/0911.2841

[10] L. Fatibene, M.Ferraris, M.Francaviglia, S.Mercadante, Further Extended Theories of Gravitation: Part II, Int. J. Geom. Methods Mod. Phys. Volume: 7, Issue: 5 (2010), pp. 899-906; gr-qc/0911.284

[11] N. Dadhich, J.M. Pons, Equivalence of the Einstein-Hilbert and the Einstein-Palatini formulations of general relativity for an arbitrary connection, (to appear on GRG); arXiv:1010.0869v3 [gr-qc]

[12] C. Rovelli, What is observable in classical and quantum gravity? Class. Quantum Grav. 8: 297, 1991;

[13] L. Fatibene, M. Francaviglia, Weyl Geometries and Timelike Geodesics, arXiv:1106.1961v1 [gr-qc]

[14] L. Fatibene, M. Francaviglia, Fluids in Weyl Geometries, arXiv:1109.4115v1 [math-ph]

[15] M.Ferraris, J.Kijowski, Unified geometric theory of electromagnetic and gravitational interactions GRG 14(1) (1982), 37-47 